\title{
Zinc: A complementary factor in the treatment of chronic hepatitis C? (Review)
}

\author{
KURT GRÜNGREIFF $^{1}$ and DIRK REINHOLD ${ }^{2}$ \\ ${ }^{1}$ Heydeckstrasse 9, 39104 Magdeburg; ${ }^{2}$ Institute of Molecular and Clinical Immunology, \\ Otto von Guericke University Magdeburg, Leipziger Strasse 44, 39120 Magdeburg, Germany
}

Received January 26, 2010; Accepted March 2, 2010

DOI: 10.3892/mmr_00000267

\begin{abstract}
Chronic hepatitis C (HCV) infection persists in more than 170 million people worldwide and is one of the major causes of hepatic failure and liver transplantation. Current treatment of chronic $\mathrm{HCV}$, consisting of pegylated interferon and ribavirin, is associated with a wide range of side effects, contraindications and costs, and leads to viral clearance in only $50-55 \%$ (genotype 1) to $80 \%$ (genotype 3 ) of cases. Thus, the development of more efficient treatment regimes with fewer side effects and costs is of high priority. It is generally accepted that the cellular immune response plays the most important role in determining the outcome of $\mathrm{HCV}$ infection. Moreover, oxidative stress is considered to be an important pathogenic factor. Zinc is an essential nutrient for a broad range of biological activities. It is necessary for normal liver function, and vice versa the liver plays a central role in zinc homeostasis. Zinc ions are crucial for multiple aspects of the immune system, including the normal development, differentiation and function of cells belonging to both innate and acquired immunity. Among the immune cells that are affected by zinc deficiency, T lymphocytes are noted to have the highest susceptibility. Zinc deficiency causes substantial impairment of cellular immunity, oxidation and damage to DNA. Several studies have investigated the effect of zinc supplementation in chronic $\mathrm{HCV}$ patients. Following zinc supplementation, decreases in the incidence of gastrointestinal disturbances, body weight loss and hair loss were found in patients with chronic HCV, along with improved fingernail health. In addition, zinc administered in combination with IFN- $\alpha$ was more effective against chronic HCV than treatment with IFN- $\alpha$ alone. Finally, in addition to the effects of zinc on immune functions and viral defence, its role as an antioxidant may be important in HCV. To conclude, the controlled application of zinc, particularly in a deficient state, is recommended as a complementary therapy for chronic hepatitis $\mathrm{C}$.
\end{abstract}

Correspondence to: Dr Kurt Grüngreiff, Heydeckstrasse 9, 39104 Magdeburg, Germany

E-mail: dr.kgruengreiff@t-online.de

Key words: zinc, hepatitis C

\section{Contents}

1. Introduction

2. Immunopathogenesis of chronic $\mathrm{HCV}$

3. Zinc in the liver and in the immune system

4. Oxidative stress: Chronic HCV and zinc supplementation

5. Conclusion

\section{Introduction}

Hepatitis C virus (HCV) infection becomes chronic in 70-80\% of cases, and cirrhosis of the liver ensues in $20-25 \%$ of cases. The risk of developing hepatocellular carcinoma is also increased. Worldwide, at least 100 million people are infected with this virus. In Germany, almost 800,000 people are thought to be infected. The fact that the disease often remains asymptomatic for many years means that there is a risk of underestimating the severity of the disease. The primary objective of antiviral treatment of chronic hepatitis $\mathrm{C}$ is the absence of hepatitis C-specific RNA in the serum 6 months after the end of treatment, as confirmed by polymerase chain reaction.

Over the past 15 years, a significant improvement in the response rate has been achieved. The sustained response rate with interferon- $\alpha$ (IFN- $\alpha$ ) monotherapy was $15 \%$, whereas, with a combination of IFN- $\alpha$ and ribavirin, a response rate of approximately $40 \%$ was achieved. Following the introduction of pegylated interferon (PEG-IFN), which in contrast to IFN- $\alpha$ only needs to be injected once a week, efficient levels remained unaltered for 7 days. Today, with the combination of PEG-IFN and ribavirin, a sustained response rate of $60 \%$ is achieved (1).

For both patients and doctors, it is particularly frustrating that, despite the clear progress that has been made, roughly $50 \%$ of patients treated still either do not respond to treatment or suffer a relapse. This fact is driving the continued search for new substances and mechanisms of action capable of increasing the efficacy of treatment.

The precise mechanism of liver cell damage and the chronification of HCV infections has not yet been clearly identified. Existing experimental and clinical data suggest immunologically mediated destructive mechanisms, rather than direct cytotoxic effects, leading to hepatocellular damage. 


\section{Immunopathogenesis of chronic $\mathrm{HCV}$}

Current data suggest that HCV uses various complex strategies to subvert the congenital and acquired immune response of the infected individual (2). The immune response in the case of chronic HCV is a double-edged sword. Even when $\mathrm{HCV}$ has not been effectively eliminated in the acute phase of the HCV infection, the immunological effector mechanisms are able to mediate liver damage in chronic $\mathrm{HCV}$ infection.

A defective antiviral immune response causes continued activation of proinflammatory cytokine secretion and unspecific effector mechanisms. These lead to lasting damage to hepatocytes, to fibrogenesis, and to malign transformation (2). HCV persistence is at least partly linked to an insufficient $\mathrm{CD} 4^{+}$and $\mathrm{CD} 8^{+}$cell response (3). Although almost all patients produce antibodies against the virus, and although it is frequently the case that cellular immune reactions are shown to occur, it is often not possible to eliminate the pathogen. The immune responses of patients with resolved infections and patients with chronic viremia often exhibit significant differences. In patients with a resolved HCV infection, T helper cells are more easily stimulated, and there are a larger number of antigens than is the case in patients with chronic hepatitis C. The humoral immune response, however, is stronger in patients with chronic HCV. It seems that the activation of the cellular immune system is more important than the humoral response as far as the capacity to overcome the HCV infection is concerned. The imbalance between cellular and humoral immunity in chronic HCV is due in part to an imbalance between Th1 and Th2 cytokines. Th1 cytokines [IL-2, interferon- $\gamma$ (IFN- $\gamma$ ), TNF- $\alpha$ ] support cellular effector mechanisms, whereas Th2 cytokines (IL-4, IL-10) support the production of antibodies while simultaneously inhibiting cellular mechanisms. Factors which support the production of Th1 cytokines aid virus elimination significantly. Ultimately, an insufficient $\mathrm{T}$ cell immune response can be seen as the main cause of the chronification of an HCV infection (4).

Recent studies have shown that endogenous factors such as regulatory $\mathrm{T}$ cells (Treg), immune-suppressive cytokines and inhibiting receptors help to restrict the virus-specific T-cell response, as is the case with chronic hepatitis B viral infection (HBV) and HCV infection $(5,6)$. This reflects the efforts of the infected organism to protect itself against immune-mediated pathological changes.

Factors of this type, which lead to a functional modulation of monocytes and macrophages over the course of a chronic $\mathrm{HCV}$ infection, include IFN- $\gamma$, endotoxin (lipopolysaccharide, LPS) and the HCV core protein (7). A key role in the innate immune system is played by toll-like receptors (TLRs). These receptors can recognise not only bacterial LPSs and lipoproteins, but also viral antigens and nucleic acid. However, they do not only stimulate unspecific immune mechanisms, but are also important for the production of specific immune reactions and the immunological memory.

Thus dendritic cells (DCs), which synthesise TLRs, can activate B-T lymphocytes. TLRs are also closely involved in zinc homeostasis and the activation of DCs, which play an important role in the clearance of pathogenic structures (8).
Stimulation with the TLR4 agonist LPS restricts the expression of zinc transporters into the DCs, thus leading to a reduction in free intracellular zinc. Jarousse et al (9) discovered that zinc salts in keratinocytes inhibited TLR2 expression. As far as we are aware, TLRs play an important role in the pathogenesis of various liver diseases, including non-alcoholic steatohepatitis, autoimmune hepatitis, primary biliary cirrhosis and chronic hepatitis B and C. Initial studies are being carried out to test the potential of TLR agonists in the treatment of these diseases (10).

\section{Zinc in the liver and in the immune system}

Zinc plays a key role in numerous biochemical and physiological processes. It is a component of more than 150 enzymes, and has a catalytic effect due to its direct involvement in substrate conversion and the stabilisation of the enzyme structure. Zinc has a structural effect on transcription factors and a regulatory effect on hormones, hormone receptors and gene expression. Its influence on metabolism, growth and development has been proven (11). Zinc plays an important role as a second messenger and signal molecule, and is crucial to the redox process. The cell-damaging oxidative stress that can be caused by a zinc deficiency is a fundamental principle (11). Approximately $10 \%$ of the zinc in the body is metabolically active. The liver is the main organ involved in zinc metabolism. Zinc is present in the liver in both a form that can be rapidly mobilised and in a form that can only slowly be mobilised. The regulatory processes are predominantly hormonally controlled by insulin, glucagon and glucocorticoids. Depending on the specific situation, these substances lead to a transient dysregulation in zinc metabolism in the liver cells, with subsequent plasma zinc deficiency. Mediator substances have similar effects. There is close interaction between metallothionein (MT), an acute-phase protein, and zinc. On the one hand, MT plays an important role in the resorption, distribution and cellular accumulation of zinc, while on the other, the increased consumption of zinc leads to increased MT synthesis.

Close interaction has also been shown to exist between zinc and IL-6, an important proinflammatory cytokine for the regulation of the acute-phase gene. The action of zinc in the hepatic synthesis of acute-phase proteins, in the regulation of gluconeogenesis, in controlling reactive substances (e.g. nitrogen oxide) or hydrophilic radicals, and in controlling microbiological growth, are all functions of IL-6. It regulates the zinc transporter Zip 14 in the liver, thus contributing to hypozincemia in the acute-phase reaction. Tracer studies have shown that a complete zinc exchange occurs in the hepatocytes in less than two days. A reduction in the amount of zinc in the liver leads to a reduction in the regenerative capacity of hepatocytes.

Changes in zinc status have a direct altering effect on gene expression. mRNA levels of MT, cholecystokinin, uroguanylin, endothelin and retinol-binding protein rise and fall as a consequence of changes in zinc status. A zinc deficiency therefore influences various hepatic functions and, because of the importance of the liver in the body, zinc plays a key role in numerous biochemical and physiological processes. Zinc deficiency has an effect on the production of thyroid 


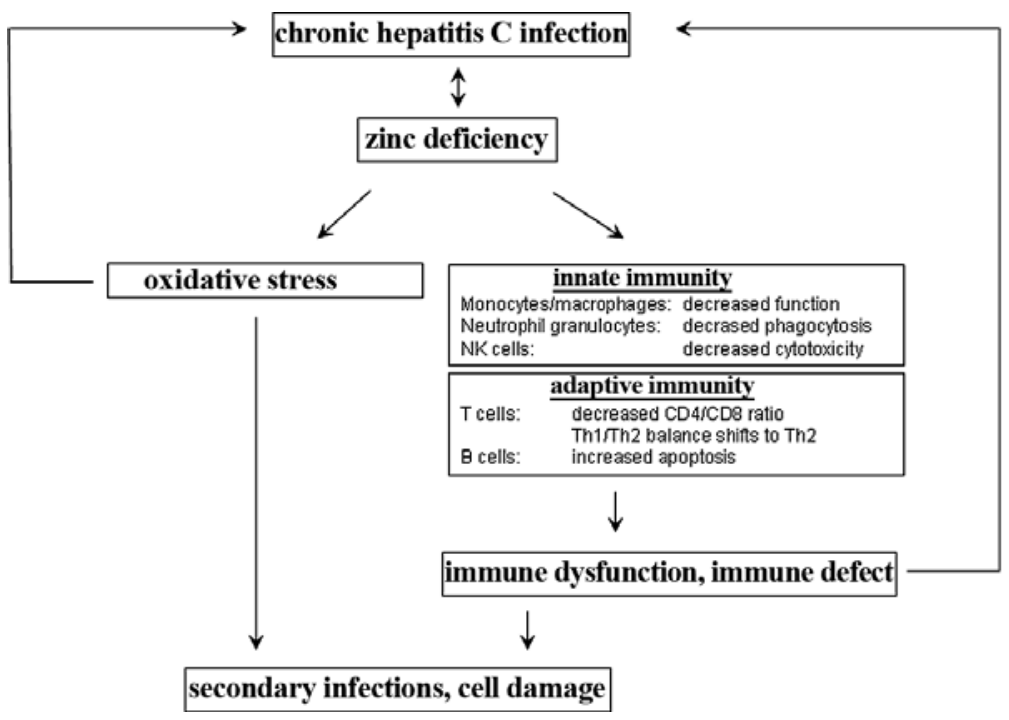

Figure 1. Interaction between zinc homeostasis and chronic hepatitis $\mathrm{C}$ infection.

hormones, the hepatic lipid metabolism, and the effectiveness of the cytochrome P 450 enzyme (reviewed in ref. 12). In the past few years it has become clear that there is a close relationship between the trace element zinc and the immune system. Zinc deficiency in humans and artificially created zinc deficiency in animals are frequently accompanied by a defective immune system. In most cases, zinc therapy normalises these immune defects. Low zinc concentrations have a stimulating effect on various functional parameters of immune cells in vitro, while higher zinc concentrations are cytotoxic for these cell populations. The fact that zinc is involved in the regulation of more than 150 enzymes means that it plays an important role in cellular and humoral immunity (Fig. 1). Zinc influences $\mathrm{T}$ and $\mathrm{B}$ lymphocytes as well as NK cells and monocytes (13-15). It performs special tasks in the antigen-specific immune response ( $\mathrm{T}$ lymphocytedependent cellular immunity and antibody response via antigen-stimulated B lymphocytes) and in unspecific immune mechanisms (phagocytosis, complement activation) that cannot be performed by any other trace element, which gives zinc a certain exclusivity. In this way, zinc influences, among other things, the binding of the signal transduction element p56 $6^{\text {Ick }}$ to $\mathrm{CD}^{+}$or $\mathrm{CD}^{+}$, and is therefore essential for $\mathrm{T}$ cell activation (16). By binding to the NK cell receptor 'killer cellinhibitory D receptor (KIR2D)', zinc regulates the activity of NK cells (17). Zinc deficiency has, above all, an adverse effect on $\mathrm{T}$ cells, leading to reduced numbers of $\mathrm{T}$ cells and to the defective functioning of $\mathrm{T}$ cells, including a shift in the Th cell response to Th2 predominance (18). In consideration of the essential role of zinc for effective $\mathrm{T}$ cell function, zinc deficiency could therefore be significant in terms of the pathogenesis of chronic $\mathrm{HCV}$, and possibly also in terms of the success of a course of antiviral treatment (Fig. 1).

In our own experience, $40 \%$ of patients with chronic hepatitis $\mathrm{C}$ are shown to have a zinc deficiency that is aggravated during antiviral treatment. Up to $75 \%$ of patients with liver cirrhosis are at least temporarily deficient in zinc (19). Many years of observation have shown that serum zinc concentrations decrease in the course of treatment of chronic HCV with
Table I. Possible effects of zinc in patients with chronic hepatitis $\mathrm{C}$.

Regulation of the imbalance between Th1 and Th2 cells.

Radical-scavenger function and reduction in the side effects of IFN- $\alpha$.

Hepatoprotective effects through induction of metallothionein synthesis.

Activation of the IFN receptor through stimulation of dimerisation of IFN.

Zinc boosts the antiviral effect of IFN- $\alpha$ 10-fold.

Inhibitory effect of zinc on HCV replication.

PEG-IFN and ribavirin, especially in the case of responders. One possible explanation for this could be the increased zinc consumption involved in cytokine-induced immunological processes. This explanation is supported by the fact that IL-6 plasma concentrations, which were are found to be raised at the beginning of treatment, are normalised at the end of the course of treatment. Zinc levels can be normalised by the temporary administration of a zinc supplement. The symptoms which accompany antiviral treatment, such as hair loss, dry skin and brittle nails (symptoms of zinc deficiency), can be partially or entirely alleviated by administering zinc (20). These observations, made on the basis of our own experience of individual cases, are confirmed by the results of a randomised study by Takagi et al (21), which showed a higher response rate for a combination of IFN- $\alpha$ plus zinc compared to IFN- $\alpha$ alone.

\section{Oxidative stress: Chronic HCV and zinc supplementation}

Alongside the insufficient $\mathrm{T}$ cell functions, increasing importance is being attached to the presence and action of oxidative stress in patients with $\mathrm{HCV}$ as a factor leading to the chronification of the disease $(22,23)$. It is assumed that the hepatic oxidative stress induced by $\mathrm{HCV}$ is, on the one hand, a direct consequence of the virally triggered destruction of mitochondria and other cell organelles of the hepatocytes, and, on the 
other, that it leads to the initiation of an inflammatory reaction in the liver. Alongside the direct effect of the HCV core protein, hepatocellular iron accumulation and the production of ROS associated with the immune response are considered to be of crucial significance for the creation of oxidative stress in chronic HCV $(23,24)$. Zinc plays an important role in the redox process as a signal molecule and second messenger. Disruption of zinc homeostasis can contribute to the chronification of various diseases. Cell damaging oxidative stress, which can be induced by zinc deficiency, is a fundamental principle (11). Not only a diet-related zinc deficiency but also a conditioned zinc deficiency caused by the effect of environmental chemicals or medicines (including PEG-IFN) should be considered as a cause of the disease (25). Studies by Murakami et al (26) showed that zinc substitution in patients with chronic $\mathrm{HCV}$ results in the induction of antioxidative functions in the liver, which leads to decreased liver damage during treatment with peginterferon and ribavirin. Himoto et al (27) recommend the administration of zinc supplements for patients with chronic hepatitis $\mathrm{C}$, as zinc significantly lowers the disease activity by lowering iron concentration. Nuclear factor $(\mathrm{NF})-\kappa \mathrm{B}$ is considered to be an important factor in the initiation of oxidative stress in acute inflammatory processes, particularly in the liver, as a consequence of viral, metabolic and immunological genesis (28). To date, it has not been possible to conclusively assess the role of $\mathrm{NF}-\kappa \mathrm{B}$ in the persistence of chronic HBV or HCV infection, as there are no reliable data from primates or humans (28). Irrespective of this, it has been possible to prove that zinc substitution leads to the reduction of inflammatory cytokines as it inhibits $\kappa \mathrm{B}$ phosphorylation and NF- $\kappa \mathrm{B}$ activation (29).

Recent studies by Yuasa et al (30) have shown that zinc substitution negatively influences $\mathrm{HCV}$ replication. The mechanisms behind this inhibiting influence on the HCV genome in HCV-RNA replicating cells have not yet been clearly identified. Observations in clinical experience of the positive influence of zinc substitution in a large proportion of patients receiving treatment for chronic hepatitis $\mathrm{C}$ may thus also be backed up by experimental studies. The proven and assumed effects of zinc in the treatment of chronic viral infections, including hepatitis $\mathrm{C}$, are produced via immunological reactions, antiviral defence mechanisms and the role of zinc as an antioxidant (31).

\section{Conclusion}

It can be concluded that understanding the interaction between innate and acquired immunity and the hepatitis $\mathrm{C}$ virus is essential for the development of new therapeutic strategies in the fight against this disease. Although few studies have been carried out on the use of zinc in the treatment of chronic $\mathrm{HCV}$, it seems justified to administer a zinc supplement in addition to the current standard treatment with PEG-IFN and ribavirin, at least where a zinc deficiency in the serum has been detected, especially as this supplement is inexpensive and is associated with only minimal side effects. On the basis of many years of experience, we would recommend zinc substitution in patients with a proven zinc deficiency (with a zinc level in the serum of $<10 \mu \mathrm{mol} / \mathrm{l}: 15 \mathrm{mg} / \mathrm{day}$; with a level of $<8 \mu \mathrm{mol} / \mathrm{l}: 30 \mathrm{mg} /$ day). The patient should be monitored after $6-8$ weeks. Once zinc concentrations have normalised, administration of the supplement can be discontinued (32). Possible points of action of a zinc supplement in chronic hepatitis $\mathrm{C}$ are shown in Table I.

\section{References}

1. Manns MP, Wedemeyer $\mathrm{H}$ and Cornberg M: Treating viral hepatitis C: efficacy, side effects and complications. Gut 55: 1350-1359, 2006

2. Spengler U and Nattermann J: Immunopathogenesis of hepatitis C cirrhosis. Clin Sci 12: 141-155, 2007.

3. Bowen DG and Walker CM: Adaptive immune responses in acute and chronic hepatitis virus infection. Nature 436: 946-952, 2005.

4. Jacobson Brown PM and Neuman MG: Immunopathogenesis of hepatitis $\mathrm{C}$ viral infection: Th1/Th2 responses and the role of cytokines. Clin Biochem 34: 167-171, 2001.

5. Rehermann B: Chronic infections with hepatotropic viruses: Mechanisms of impairment of cellular immune responses. Sem Liver Dis 27: 152-160, 2007.

6. Dolganiuc A and Szabo G: T cells with regulatory activity in hepatitis $\mathrm{C}$ virus infection. What we can know and what we don't. J Leukoc Biol 84: 614-622, 2008.

7. Dolganiuc A, Norkina O, Kodys K, Catalano D, Bakis G, Marshall C, Mandrekar P and Szabo G: Viral and host factors induce macrophage activation and loss of toll-like receptor tolerance in chronic HCV infection. Gastroenterology 133: 1627-1636, 2007.

8. Kitamura H, Morikawa H, Kamon H, et al: Toll-like receptormediated regulation of zinc homeostasis influences dendritic cell function. Nat Immunol 7: 971-977, 2006.

9. Jarousse V, Castex-Rizzi N, Khammari A, Charveron M and Dreno B: Zinc salts inhibit in vitro Toll-like receptor 2 surface expression by keratinocytes. Eur J Dermatol 17: 492-496, 2007.

10. Seki E and Brenner D: Toll-like receptors and adaptor molecules in liver disease: update. Hepatology 48: 322-335, 2008.

11. Maret W: Zinc and sulfur: A critical biological partnership. Biochemistry 4: 3301-3309, 2004.

12. Grüngreiff K and Reinhold D: Zink: Bedeutung in der ärztlichen Praxis. J. Hartmann Verlag, Hessdorf-Klebheim, 2007.

13. Wellinghausen N, Kirchner H and Rink L: The immunbiology of zinc. Immunol Today 18: 519-521, 1997.

14. Rink L and Kirchner H: Zinc altered immune function and cytokine production. J Nutr 130: 1407S-1411S, 2000.

15. Reinhold D, Ansorge $S$ and Grüngreiff $\mathrm{K}$ : Zinc regulates DNA synthesis on IL-2, IL-6, and IL-10 production of PWM-stimulated PBMC and normalizes the peripheral cytokine concentration in chronic liver disease. J Trace Elem Exp Med 10: 19-27, 1997.

16. Lin RS, Rodriguez C, Veillette A and Lodish HF: Zinc is essential for binding of p561ck to CD4 and CD8alpha. J Biol Chem 273: 32878-32882, 1998.

17. Rajagopalan S and Long EO: Zinc bound to the killer cellinhibitory receptor modulates the negative signal in human NK cells. J Immunol 161: 1299-1305, 1998.

18. Rink L and Haase H: Zinc homeostasis and immunity. Trends Immunol 28: 1-4, 2007.

19. Grüngreiff K: Betreuung Hepatitiskranker in der Praxis. Med Welt 58: 251-258, 2007.

20. Grüngreiff K, Reinhold D and Ansorge S: Serum concentrations of sIL-2, IL-6, TGF- $\beta 1$, neopterin, and zinc in chronic hepatitis $\mathrm{C}$ patients treated with interferon-alpha. Cytokine 11: 1076-1080, 1999.

21. Tagaki H, Nagamine T, Abe T, et al: Zinc supplementation enhances the response to interferon therapy in patients with chronic hepatitis C. J Viral Hepat 8: 367-371, 2001.

22. Beard MR and Jones BE: Hepatitis $C$ virus and oxidative stress: a dangerous liaison. Future Virol 1: 223-232, 2006.

23. Wang T and Weinman SA: Causes and consequences of mitochondrial reactive oxygen species generation in hepatitis C. J Gastroenterol Hepatol 21: S34-S37, 2006.

24. Okuda M, Li K, Beard MR, Showalter LA, Scholle F, Lemon SM and Weinman SA: Mitochondrial injury, oxidative stress, and antioxidant gene expression are induced by hepatitis $\mathrm{C}$ virus core protein. Gastroenterology 122: 366-375, 2002.

25. Maret W and Krezel A: Cellular zinc and redox buffering capacity of metallothionein/thionein in health and disease. Mol Med 13: 371-375, 2007. 
26. Murakami Y, Koyabu T, Kawashima A, et al: Zinc supplementation prevents the increase of transaminase in chronic hepatitis $\mathrm{C}$ patients during combination therapy with pegylated interferon alpha-2b and ribavirin. J Nutr Sci Vitaminol 53: 213-218, 2007.

27. Himoto T, Hosomi N, Nakai S, et al: Efficacy of zinc administration in patients with hepatitis $\mathrm{C}$ virus-related chronic liver disease. Scand J Gastroenterol 42: 1078-1087, 2007.

28. Sun B and Karin M: NF- $\mathrm{BB}$ signaling, liver disease and hepatoprotective agents. Oncogene 27: 6228-6244, 2008.

29. Prasad AS: Zinc. Mechanisms of host defense. J Nutr 137: 1345-1349, 2007.
30. Yuasa K, Naganuma A, Sato K, Ikeda M, Kato N, Takagi H and Mori M: Zinc is a negative regulator of hepatitis $\mathrm{C}$ virus RNA replication. Liver Int 26: 1111-1118, 2006.

31. Overbeck S, Rink L and Haase H: Modulating immune response by oral zinc supplementation. Arch Immunol Ther Exp 56: 15-30, 2008.

32. Grüngreiff K: Zink - ein Adjuvans in der chronischen Hepatitis B. Hep Net J 1: 12-13, 2007. 